\title{
Pressure-temperature phase diagram of the generalized Hubbard model with correlated hopping at half-filling
}

\author{
L.Didukh, V.Hankevych* \\ Ternopil State Technical University, Department of Physics, \\ 56 Rus'ka Str., 46001 Ternopil, Ukraine
}

Received August 14, 2000

\begin{abstract}
In the present paper, the pressure-temperature phase diagram of a generalized Hubbard model with correlated hopping in a paramagnetic state at half-filling is determined by means of a generalized mean-field approximation in the Green function technique. The constructed phase diagram describes the metal-to-insulator transition with increasing temperature, and the insulator-to-metal transition under the action of external pressure. The phase diagram can explain the paramagnetic region of the phase diagrams of some transition metal compounds.
\end{abstract}

Key words: phase diagram, metal-insulator transition, correlated hopping

PACS: $71.10 . F d, 71.30 .+h, 71.27 .+a$

\section{Introduction}

In recent years a generalized Hubbard model with correlated hopping has been used widely to describe strongly correlated electron systems (see papers $[1,2]$ and references therein); the electron-hole asymmetry is a property of such a generalized Hubbard model as a result of the dependence of the hopping integral on the occupation of the sites involved in the hopping process. Recently, this model has been extended to the case of a doubly orbitally degenerate band [3].

The generalized Hubbard model has much richer properties than the well-known Hubbard model [4], and usage of the electron-hole asymmetry concept allows one to interpret the peculiarities of physical properties of narrow-band materials which are not explained by the Hubbard model. In particular, the experimentally observed electron-hole asymmetries of metal oxides conductivity, of cohesive energy of transition $3 d$-metals and of superconducting properties of high-temperature superconductors have been explained within the generalized Hubbard model with correlated hopping in papers [5-10] respectively.

*E-mail: vaha@tu.edu.te.ua 
Despite the fact that the phase diagram of the generalized Hubbard model has been studied in works [11-17], researchers pay no attention to determining the model phase diagram in a paramagnetic state under the action of external effects, in particular the pressure-temperature phase diagram. This task is related directly to the problem of the metal-insulator transition description under the action of external pressure and temperature, namely the constructed pressure-temperature phase diagram of the model would allow us to describe the observed metal-insulator transitions in narrow-band materials with the change of pressure and temperature. An interest to such transitions is caused by the theoretical point of view as well as by the rich possibilities of its application (see, for example, monograph [18] and review [19]). Consequently, the goal of the present paper, being a continuation of the previous work [20] where the temperature-induced metal-insulator transition was studied, is to determine the pressure-temperature phase diagram of the generalized Hubbard model with correlated hopping in a paramagnetic state at half-filling. Based on this phase diagram, we describe the metal-insulator transitions under the action of external pressure and temperature.

\section{Pressure-temperature phase diagram of the model}

Taking into account an external hydrostatic pressure $p$ we write the model Hamiltonian in the following form [5] (in this connection also see [21]):

$$
\begin{aligned}
H= & -\mu \sum_{i \sigma} a_{i \sigma}^{+} a_{i \sigma}+(1+\alpha u) t \sum_{i j \sigma}^{\prime} a_{i \sigma}^{+} a_{j \sigma}+T_{2} \sum_{i j \sigma}^{\prime}\left(a_{i \sigma}^{+} a_{j \sigma} n_{i \bar{\sigma}}+\text { h.c. }\right) \\
& +U \sum_{i} n_{i \uparrow} n_{i \downarrow}+\frac{1}{2} N V_{0} \kappa u^{2},
\end{aligned}
$$

where $i, j$ are the nearest-neighbours sites, $\mu$ is the chemical potential, $a_{i \sigma}^{+},\left(a_{i \sigma}\right)$ is the creation (destruction) operator of an electron of spin $\sigma(\sigma=\uparrow, \downarrow)$ on site $i(\bar{\sigma}$ denotes spin projection which is opposite to $\sigma), n_{i \sigma}=a_{i \sigma}^{+} a_{i \sigma}$ is the number operator of electrons of spin $\sigma$ on site $i, U$ is the intra-atomic Coulomb repulsion, $t=t_{0}+T_{1}$, with $t_{0}$ being the matrix element of the electron-ion interaction, $T_{1}, T_{2}$ are the correlated hopping integrals (matrix elements of electron-electron interaction), the primes on the sums in Hamiltonian (2.1) signify that $i \neq j$.

The last term of the Hamiltonian has the meaning of an elastic energy of a uniformly deformed crystal, where $\kappa$ is the "initial" (purely lattice) bulk elasticity, $N$ is the number of lattice sites, $u=\Delta V_{0} / V_{0}$ is the relative change of the volume in uniform strain ( $V_{0}$ is the initial unit-cell volume). Formulating the Hamiltonian we have used the result of paper [21]: the dependence of a bandwidth $W$ on relative change of the volume $u$ in uniform strain can be written in the form $W=2 w(1+\alpha u)$, where $w=z|t|$ ( $z$ is the number of the nearest neighbours to a site), $\alpha=\frac{V_{0}}{2 w} \frac{\partial W}{\partial V}<0$. We also assume that under the action of external pressure only the bandwidth changes, and the matrix elements of the electron-electron interaction (the correlated hopping integrals and intra-atomic Coulomb repulsion) do not depend on the relative change of the volume. 
As in papers $[2,20]$, using the generalized mean-field approximation [5,22] (an analog of the projection operation) in the Green function method, we obtain for a paramagnetic state at half-filling the energy gap width as

$$
\begin{aligned}
& \Delta E=-(1-2 d)(w+\tilde{w})[1+\alpha u]+\frac{1}{2}\left(Q_{1}+Q_{2}\right), \\
& Q_{1}=\sqrt{[B(w-\tilde{w})(1+\alpha u)-U]^{2}+\left[4 d z t^{\prime}(1+\alpha u)\right]^{2}}, \\
& Q_{2}=\sqrt{[B(w-\tilde{w})(1+\alpha u)+U]^{2}+\left[4 d z t^{\prime}(1+\alpha u)\right]^{2}},
\end{aligned}
$$

where $B=1-2 d+4 d^{2}, d$ is the concentration of polar states (holes or doublons) which has been calculated in $[2,20], \tilde{w}=z|\tilde{t}|, \tilde{t}=t+2 T_{2}, t^{\prime}=t+T_{2} ; t$ and $\tilde{t}$ are the terms describing hopping of quasiparticles within the lower and upper Hubbard bands (hopping of holes and doublons) respectively, $t^{\prime}$ describes a quasiparticle hopping between hole and doublon bands (the processes of paired creation and destruction of holes and doublons).

According to the method proposed for the s(d)-f model in paper [23], the equilibrium value of relative change of the volume $u$ is determined from the condition of the minimum of the thermodynamic Gibbs' potential

$$
G=F+N p V_{0}(1+u)
$$

where $F$ is the free energy. Using the known identity $\partial F / \partial u=\langle\partial H / \partial u\rangle$, equation (2.5) for the parameter $u$ can be represented as

$$
\left\langle\frac{\partial H}{\partial u}\right\rangle+N p V_{0}=0
$$

with $H$ being Hamiltonian (2.1). In the mean-field approximation passing to the space of quasi-momenta we get the following equation for the relative change of the volume $u$ :

$$
\alpha u=\frac{2 \alpha_{1} V_{0}}{W N} \sum_{\mathbf{k} \sigma} t_{\mathbf{k}}\left\langle a_{\mathbf{k} \sigma}^{+} a_{\mathbf{k} \sigma}\right\rangle+\tau p V_{0},
$$

where $\alpha_{1}$ is the parameter which determines the quantity $\partial W / \partial V, 2 \alpha_{1} V_{0} / W \approx$ $0.1, \tau \approx 0.05 \mathrm{eV}^{-1}[21]$

Taking into consideration the fact that within the generalized mean-field approximation the first term of the right-hand side of equation (2.7) is equal to zero [24] at the point of the metal-insulator transition, we obtain the relation between the relative change of the volume $u$ and an external hydrostatic pressure $p$ as

$$
\alpha u=\tau p V_{0}
$$

Note that within the generalized Hartree-Fock approximation this equation is valid at the point of the metal-insulator transition as well as in an insulating phase.

To determine the pressure-temperature phase diagram of the model we use formula (2.2) for the energy gap width and the expression for the concentration of 
polar states calculated in [20]. Let us consider, for instance, the Mott-Hubbard compound $\mathrm{NiS}_{2}$. This has two electrons half filling an $e_{g}$ band, the half-width of the initial (uncorrelated) band of this crystal is $w_{0}=z\left|t_{0}\right| \approx 1.05 \mathrm{eV}[26,27]$, and the initial unit-cell volume is $V_{0} \approx 14.79 \cdot 10^{-30} \mathrm{~m}^{3}$ [28]. It shows the transition from the state of a paramagnetic insulator to the paramagnetic metal state at a hydrostatic external pressure of $46 \mathrm{kbar}$ and room temperatures. Thus the transition occurs for a decrease in volume of about $0.4 \%$ with no change in crystal structure $[29,30]$. It also becomes metallic on alloying with $\mathrm{Ni}_{2} \mathrm{Se}$, and the behaviour of this system is discussed later in this section.

To calculate the model parameter $U$ we fix one of the points ( $p=22 \mathrm{kbar}$, $T=100 \mathrm{~K}$ ) of the experimental curve in the phase diagram (the dashed-line curve of figure 1) and find the value of intra-atomic Coulomb repulsion $U$ at which the theoretical calculations within the present model reproduce this point. Thus, we obtain: $U / w=U / w_{0}=2.0168$ for the correlated hopping parameters $\tau_{1}=T_{1} /\left|t_{0}\right|=$ $0, \tau_{2}=T_{2} /\left|t_{0}\right|=0$ (these values of $\tau_{1}, \tau_{2}$ correspond to the Hubbard model), $U / w=U / w_{0}\left(1-\tau_{1}\right)=1.79107$ for $\tau_{1}=\tau_{2}=0.1$, and $U / w=U / w_{0}=1.81437$ at $\tau_{1}=0, \tau_{2}=0.1$. Using these values of the model parameters we find the values of external hydrostatic pressure and temperature at which the energy gap width is equal to zero (i.e. metal-insulator transition occurs).

The calculated pressure-temperature phase diagram of the model (figure 1) describes metal-insulator transitions in a paramagnetic state under the action of an external pressure and temperature in $\mathrm{NiS}_{2}$, namely the constructed phase diagram describes the metal-to-insulator transition with increasing temperature, and the insulator-to-metal transition under the action of external pressure. Comparison of this theoretically determined phase diagram with the phase diagram of the compound $\mathrm{NiS}_{2}$ shows good agreement between the theory and experiment. Besides, the theoretical calculations within the model reproduce the experimental data of paper [29] which point out the presence of an energy gap width $\Delta E>0$ in the ground state of $\mathrm{NiS}_{2}$ and in the absence of an external pressure. The phase diagram shows that our taking into account the correlated hopping permits a much better description of

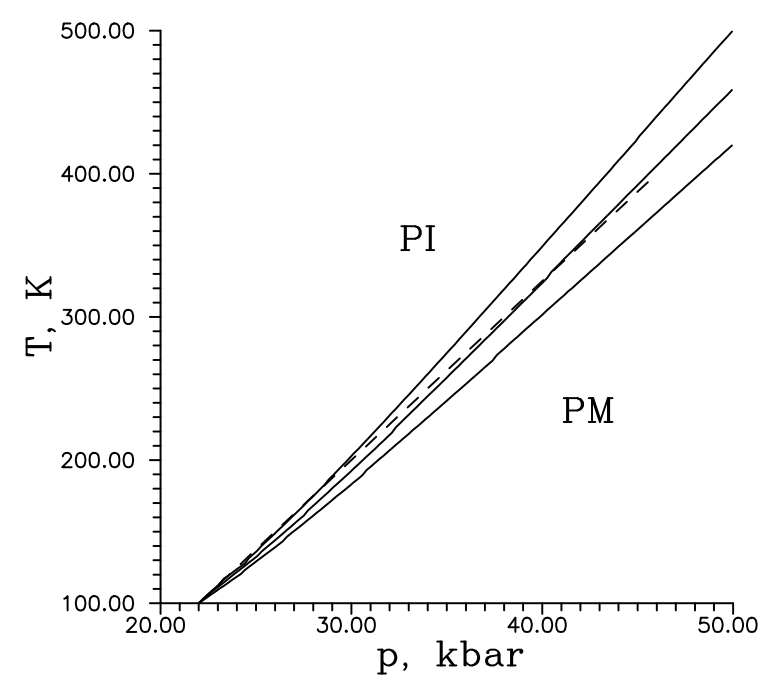

Figure 1. The pressure-temperature phase diagram of the metal-insulator transition determined within the generalized Hubbard model with correlated hopping for $\mathrm{NiS}_{2}$ in comparison with experiment (the dashed curve): $\tau_{1}=\tau_{2}=0$ (the upper curve); $\tau_{1}=0, \tau_{2}=0.1$ (the middle curve); $\tau_{1}=\tau_{2}=0.1$ (the lower curve). PI (PM) denotes paramagnetic insulating (metallic) phase. 
these experimental data than the Hubbard model; this also much better illustrates the physics of the present model and the important role of correlated hopping.

Analogous phase diagrams can be constructed for other compounds: $\left(\mathrm{V}_{1-x} \mathrm{Cr}_{x}\right)_{2} \mathrm{O}_{3}[18,25], \mathrm{NiS}_{2-x} \mathrm{Se}_{x}[26,27]$ and $\mathrm{Y}_{1-x} \mathrm{Ca}_{x} \mathrm{TiO}_{3}$ [31,32] exhibiting such metal-insulator transitions. For example, the material $\left(\mathrm{V}_{0.96} \mathrm{Cr}_{0.04}\right)_{2} \mathrm{O}_{3}$ shows a metal-insulator transition at a hydrostatic external pressure of $13 \mathrm{kbar}$ and at room temperatures; the transition occurs at a small decrease in volume of about $1 \%$ with no change in crystal structure [25]. In $\left(\mathrm{V}_{1-x} \mathrm{M}_{x}\right)_{2} \mathrm{O}_{3}$ (with $M=\mathrm{Cr}$, Ti) the addition of $\mathrm{Ti}^{3+}$ ions to $\mathrm{V}_{2} \mathrm{O}_{3}$ leads to an insulator-to-metal transition, whereas the addition of $\mathrm{Cr}^{3+}$ ions results in a metal-to-insulator transition. The simplest explanation is that the substitution of $\mathrm{V}^{3+}$ ion for $\mathrm{Cr}^{3+}$ ion leads to a band narrowing; the $\mathrm{Cr}^{3+}$ ion is a localized impurity and it deletes a state from the $3 d$-bands. Deleting a state is equivalent to a band narrowing or an external pressure decreasing [18] which drives the system towards the insulating phase. Likewise the addition of $\mathrm{Ti}^{3+}$ impurities is equivalent to an external pressure increase.

In the Mott-Hubbard compound $\mathrm{NiS}_{2-x} \mathrm{Se}_{x}$ electron hoppings between the sites of $\mathrm{Ni}$ occur through the chalcogenide sites (this is caused by peculiarities of the pyrite crystal structure [33]), the substitution of $\mathrm{S}^{2-}$ ion for $\mathrm{Se}^{2-}$ ion in $\mathrm{NiS}_{2}$ leads to an increase of wave functions overlap, consequently the probability of an electron hopping increases which is equivalent to a band broadening or to an external pressure increasing. Therefore, the pressure-temperature phase diagram constructed for $\mathrm{NiS}_{2}$ can describe the experimental composition-temperature phase diagram $[26,27]$ of the compound $\mathrm{NiS}_{2-x} \mathrm{Se}_{x}$.

Note that the doping may cause the disorder effects which can modify the electronic bands and contribute to disorder-induced localization. But for the compounds listed above, apparently, it can be assumed that the doping effects a bandwidth only or, equivalently, an effective pressure (especially for small values $x$ ). It should be also pointed out that to construct the phase diagram of the system $\mathrm{Y}_{0.61} \mathrm{Ca}_{0.39} \mathrm{TiO}_{3}$ we have to generalize the previous results obtained at half-filling to the case of a non half-filled band because this compound is characterized by such a band [32].

In conclusion, in the present paper the pressure-temperature phase diagram of the generalized Hubbard model with correlated hopping in a paramagnetic state at half-filling has been determined. The constructed phase diagram describes a metalto-insulator transition with the increasing temperature, and an insulator-to-metal transition under the action of external pressure. Comparison of this theoretically determined phase diagram with the experimental data, in particular with the phase diagram of the compound $\mathrm{NiS}_{2}$, shows good agreement between the theory and experiment. We have found that our taking into account the correlated hopping permits a much better description of these experimental data than just the Hubbard model; this also better illustrates the physics of the present model and the important role of correlated hopping.

The determined pressure-temperature phase diagram of the model can also explain the paramagnetic region of the phase diagrams of the transition metal compounds: the systems $\mathrm{NiS}_{2-x} \mathrm{Se}_{x}$ and $\left(\mathrm{V}_{1-x} \mathrm{Cr}_{x}\right)_{2} \mathrm{O}_{3}$, calcium doped $\mathrm{YTiO}_{3}$. 


\section{References}

1. Arrachea L., Aligia A.A. $d_{x^{2}-y^{2}}$ superconductivity in a generalized Hubbard model. // Phys. Rev. B, 1999, vol. 59, No. 2, p. 1333-1338.

2. Didukh L., Hankevych V. Metal-insulator transition in a generalized Hubbard model with correlated hopping at half-filling. // Phys. Stat. Sol. (b), 1999, vol. 211, No. 2, p. $703-712$.

3. Didukh L., Skorenkyy Yu., Dovhopyaty Yu., Hankevych V. Metal-insulator transition in a doubly orbitally degenerate model with correlated hopping. // Phys. Rev. B, 2000, vol. 61, No. 12 , p. $7893-7908$.

4. Hubbard J. Electron correlation in narrow energy bands. // Proc. Roy. Soc. A, 1963, vol. 276, No. 1365 , p. 238-257.

5. Didukh L. A modified form of the polar model of crystals. // Cond. Matt. Phys., 1998, vol. 1, No. 1(13), p. 125-144.

6. Didukh L., Hankevych V. On correlation effects in a narrow-band model with electronhole asymmetry. // Low Temp. Phys., 1999, vol. 25, No. 5, p. 354-358.

7. Didukh L., Hankevych V., Skorenkyy Yu. Some low-temperature properties of a generalized Hubbard model with correlated hopping. // Physica B, 2000, vol. 284-288, p. $1537-1538$.

8. Hirsch J.E. Bond-charge repulsion and hole superconductivity. // Physica C, 1989, vol. 158 , p. $326-336$.

9. Hirsch J.E. Superconductivity and hydromagnetism. // Physica B, 1990, vol. 163, p. 291-298.

10. Hirsch J.E. Hole superconductivity from kinetic energy gain. Preprint condmat/0005033, 2000, 4 p.

11. Aligia A.A., Arrachea L., Gagliano E.R. Phase diagram of extended Hubbard model with correlated hopping at half filling. // Phys. Rev. B, 1995, vol. 51, No. 19, p. 1377413777.

12. Arrachea L., Gagliano E.R., Aligia A.A. Ground-state phase diagram of an extended Hubbard chain with correlated hopping at half-filling. // Phys. Rev. B, 1997, vol. 55, No. 2, p. 1173-1184.

13. Bułka B. Superconductivity in the Hubbard model with correlated hopping: slaveboson study. // Phys. Rev. B, 1998, vol. 57, No. 17, p. 10303-10306.

14. Strack R., Vollhardt D. Hubbard model with nearest-neighbour and bond-charge interaction: exact ground-state solution in a wide range of parameters. // Phys. Rev. Lett., 1993, vol. 70, No. 17, p. 2637-2640.

15. Ovchinnikov A.A. Metal-insulator transition in the generalized Hubbard model. // J. Phys.: Condens. Matter, 1994, vol. 6, p. 11057-11069.

16. de Boer J., Schadschneider A. Exact ground states of generalized Hubbard models. // Phys. Rev. Lett., 1995, vol. 75, No. 23, p. 4298-4301.

17. Albeverio S., Fei S.-M. Some new exact ground states for generalized Hubbard models. // Europhys. Lett., 1998, vol. 41, No. 6, p. 665-670.

18. Mott N. F. Metal-insulator Transitions. London, Taylor \& Francis, 1990.

19. Imada M., Fujimori A., Tokura Y. Metal-insulator transitions. // Rev. Mod. Phys, 1998, vol. 70, No. 4, p. 1039-1264.

20. Didukh L., Hankevych V. Temperature-induced metal-insulator transition in a nonsymmetric Hubbard model at half-filling. // Condensed Matter Physics, 1999, vol. 2, 
No. 3 (19), p. 447-452.

21. Grigorchuk R.A, Stasyuk I.V. Electron-strained interaction and lattice contraction in crystals described by the Hubbard model. // Ukr. J. Phys., 1980, vol. 25, No. 3, p. 404-410 (in Russian).

22. Didukh L. Energy spectrum of electrons in the Hubbard model. // Phys. Stat. Sol. (b), 1998, vol. 206, p. R5-R6.

23. Stasyuk I.V., Grigorchuk R.A. The theory of the strain effects in the crystals described by the s(d)-f model. // Phys. Stat. Sol. (b), 1982, vol. 112, p. 327-338.

24. Didukh L., Didukh V., Dovhopyaty Yu. Metal-insulator transition in narrow energy bands of the consideration in the "spontaneous" lattice deformation. - In: Abstracts of international workshop on statistic physics. Lviv, 1995, p. 99.

25. Mc Whan D.B., Remeika J.B. Metal-insulator transition in metaloxides. // Phys. Rev. B, 1970, vol. 2, p. 3734-3739.

26. Honig J. M., Spałek J. Electronic properties of $\mathrm{NiS}_{2-x} \mathrm{Se}_{x}$ single crystals: from magnetic Mott-Hubbard insulator to normal metals. // Chem. Mater., 1998, vol. 10, No. 10, p. 2910-2929.

27. Matsuura A.Y., Shen Z.-X., Dessau D.S. et al. Electronic structure and the metalinsulator transition in $\mathrm{NiS}_{2-x} \mathrm{Se}_{x}$. // Phys. Rev. B, 1996, vol. 53, No. 12, p. R7584R7587.

28. Raybaud P., Kresse G., Hafner J., Toulhoat H. Ab initio density functional studies of transition-metal sulphides: I. Crystal structure and cohesive properties. // J. Phys.: Condens. Matter, 1997, vol. 9, No. 50, p. 11085-11106.

29. Wilson J.A., Pitt G.D. Metal-insulator transition in $\mathrm{NiS}_{2}$. // Phil. Mag., 1971, vol. 23, p. $1297-1310$.

30. Wilson J.A. The Metallic and Nonmetallic States of Matter. London, Taylor \& Francis, 1985.

31. Tokura Y., Katsufuji T. Optical spectroscopy on the Mott transition in perovskitetype titanates. - In: Spectroscopy of Mott insulators and correlated metals: proceedings of the 17th Taniguchi Symposium. Kashikojima, Japan, October 24-28, 1994 / Fujimori A., Tokura Y.- Berlin Heidelberg, Springer-Verlag, 1995, p. 163-173.

32. Iga F., Naka T., Matsumoto T. et al. Study of first-order metal-insulator transition in the strongly correlated electron system $\mathrm{Y}_{1-x} \mathrm{Ca}_{x} \mathrm{TiO}_{3}$. // Physica B, 1996, vol. 223224 , p. $526-528$.

33. Krill G., Lapierre M.F., Gautier F. et al. Electronic and magnetic properties of the pyrite-structure compound $\mathrm{NiS}_{2}$ : Influence of vacancies and copper impurities. // J. Phys. C: Solid State Phys., 1976, vol. 9, p. 761-782. 


\title{
Фазова діаграма тиск-температура узагальненої моделі Габбарда з корельованим переносом при половинному заповненні
}

\author{
Л.Дідух, В.Ганкевич
}

Тернопільський державний технічний університет імені І.Пулюя, кафедра фізики, 46001 Тернопіль, вул. Руська, 56

Отримано 14 серпня 2000 р.

Робота присвячена побудові фазової діаграми тиск-температура узагальненої моделі Габбарда 3 корельованим переносом у парамагнітному стані при половинному заповненні зони з використанням узагальненого наближення Гартрі-Фока в методі функцій Ґріна. Побудована фазова діаграма описує перехід з металічного стану в діелектричний при збільшенні температури і перехід з діелектричного стану в металічний під дією зовнішнього тиску. Фазова діаграма може пояснити парамагнітні області фазових діаграм деяких сполук перехідних металів.

Ключові слова: фазова діаграма, перехід метал-діелектрик, корельований перенос

PACS: $71.10 . F d, 71.30 .+h, 71.27 .+a$ 